\title{
AVALIAÇÃO DO USO TÓPICO DOS ÁCIDOS GRAXOS ESSENCIAIS (AGES) E OLIGOELEMENTOS NO CONTROLE DAS DERMATITES NA ÁREA DE FRALDAS EM IDOSOS
}

\author{
ASSESSMENT OF THE TOPIC USE OF ESSENTIAL FATTY ACIDS (AGES) AND \\ OLIGOELEMENTS IN THE CONTROL OF DERMATITES IN THE AGE FRALDS AREA
}

\author{
Karina Grazielle de Souza Ribeiro ${ }^{1} *$ Givanildo Carneiro Benicio $^{2} *$ Madna Avelino Silva $^{3}$ Felipe $^{*}$ \\ Lima Gadelha ${ }^{4}$
}

\begin{abstract}
RESUMO
O presente trabalho teve como objetivo avaliar o uso tópico dos Ácidos graxos essenciais (AGEs) e Oligoelementos no controle das dermatites da área das fraldas (DAF) em idosos de uma instituição de longa permanência de Fortaleza. Trata-se de um estudo descritivo, com abordagem quantitativa e delineamento transversal de pacientes idosos institucionalizados. Utilizou-se como instrumento de coleta de dados as seguintes variáveis: características demográficas, morbidades associadas, se tinha ou não incontinência urinaria ou fecal, e se faziam o uso de fraldas, além do exame físico da pele na região anogenital, nádegas, coxas e áreas adjacentes. A amostra foi composta por 27 idosos. As fórmulas farmacológicas avaliadas foram da loção oleosa (AGE-ÓLEO) em comparação ao creme Hidratante (AGE-HIDRATANTE) que contém em sua formulação Oligoelementos, que são utilizados como co-fatores na cicatrização de feridas. Os idosos foram examinados através do dermatoscópio, somado a captura de imagens avaliadas seguindo-se de elaboração de intervenções direcionadas a DAF. Em relação a presença de DAF, $(21,75 \%)$ dos idosos apresentaram alteração na pele, fazendo a terapia tópica com alguma formulação de AGE - ácidos graxos essenciais e oligoelementos associados a formulação do fármaco. Pode-se concluir que a higiene, a troca de fraldas regulares, e a hidratação adequada em conjunto com um diagnóstico precoce pela equipe de enfermagem garante através de medidas de baixa complexidade a prevenção da DAF.
\end{abstract}

Palavras-chave: Dermatite das Fraldas; Idoso; Incontinência Urinária; Incontinência Fecal.

\begin{abstract}
This study aimed to evaluate the topical use of essential fatty acids (AGEs) and trace elements in the control of diaper dermatitis (DAF) in the elderly of a long-stay institution in Fortaleza. This is a descriptive study with a quantitative approach and cross-sectional design of institutionalized elderly patients. The following variables were used as a data collection instrument: demographic characteristics, associated morbidities, whether or not there was urinary or fecal incontinence, and diapers were used, in addition to physical examination of the skin in the anogenital region, buttocks, thighs and adjacent areas. The sample consisted of 27 elderly people. The pharmacological formulas evaluated were the oily lotion (AGE-OIL) compared to the Moisturizing cream (AGE-HYDRATANT) that contains trace elements in its formulation, which are used as co-factors in wound healing. The elderly were examined using the dermoscope, added to the capture of images evaluated followed by the development of interventions aimed at DAF. Regarding the presence of DAF, $(21,75 \%)$ of the elderly presented skin changes, performing topical therapy with some formulation of EFA - essential fatty acids and trace elements associated with drug formulation. It can be concluded that hygiene, changing regular diapers, and adequate hydration together with early diagnosis by the nursing team ensure the prevention of DAF through low complexity measures.
\end{abstract}

Keywords: Diaper Dermatitis; Elderly; Urinary Incontinence; Fecal Incontinence.

\footnotetext{
${ }^{1}$ Graduada em Enfermagem pelo Centro Universitário Uninassau de Fortaleza. Pós-Graduanda em Enfermagem em UTI . Preceptora dos acadêmicos no $9^{\circ}$ semestre de Enfermagem; faz parte de dois projetos de extensão LADEPI - Liga Acadêmica de Ensino e Pesquisa em Imunologia e LAED- Liga Acadêmica de Enfermagem Dermatológica. E-mail: karinnagrazielle@ gmail.com. Orcid: https://orcid.org/0000-0001-8488-2976

${ }^{2}$ Graduado em Enfermagem pela Universidade Estadual do Piaúi Especialista em Enfermagem do trabalho e Enfermagem Dermatológica; Mestrado e Doutorado em Patologia pela Universidade Federal do Ceará. Docência do ensino superior (graduação e pós graduação); fundador e Orientador/coordenador da Liga Acadêmica de Estudos e Pesquisas da Pele; Vice Coordenador e Membro efetivo (parecerista) do Comitê de Ética e Pesquisa com seres humanos - CEP/FATENE. E-mail: givanildobenicio.c@ gmail.com Orcid: https://orcid.org/0000-0001-9642-5617

${ }^{3}$ Graduanda de Enfermagem pela Faculdade de Ensino E Cultura Do Ceara- FAECE-FAFOR. Especialista de enfermagem Unidade de Terapia Intensiva UTI pela ABEn-Ceará em Enfermagem Dermatologia pela Faculdade Estácio FIC e Gestão hospitalar. Enfermeira professora preceptora do curso de graduação de enfermagem centro universitário-UNINASSAU Fortaleza-Ce. Atuando como coordenadora cientifica da Liga Acadêmica de enfermagem Dermatológica LAED- UNINASSAU e coordenadora de enfermagem do setor de internação da Hospital Maternidade Eugenia Pinheiro HAPVIDA. Membro do associado da SOBENFeE Nacional. E-mail: madna.a.silva@ gmail.com Orcid: https://orcid.org/0000-0003-0424-4398

${ }^{4}$ Bacharel em enfermagem, pós-graduando em enfermagem do trabalho. E-mail: felipexlg@yahoo.com Orcid: https://orcid.org/0000-0002-6525-7355
} 


\section{INTRODUÇÃO}

Para fins desta pesquisa, utilizaremos o termo dermatite da área das fraldas (DAF) definido como diferentes dermatoses inflamatórias, de etiologia multifatorial, que atinge a área do corpo coberta pela fralda, afetando normalmente a região do períneo, região ano genital, região glútea, abdômen inferior e coxas. A DAF pode ser interpretada como o resultado final de simultâneos eventos e induzidas por exposição a diversos fatores, tais como: fezes, urina, hidratação, fricção, temperatura, irritantes químicos e microrganismos (1).

Uma das maiores preocupações dos enfermeiros, é prevenir lesões de pele, uma vez que o número de idosos possuidores de incontinência urinária e/ou fecal fazendo a utilização de fraldas tem se intensificado, por isso a incorporação de tecnologias provoca ao enfermeiro uma constante renovação das práticas de cuidado. Um estudo brasileiro com o objetivo de avaliar as principais tecnologias aplicadas pela enfermagem no controle urinário identificou que, $42,3 \%$ de adultos e idosos hospitalizados usavam fraldas, seguido de drenagem por cateter externo $(34,6 \%)$, cateterismo intermitente $(19,3 \%)$ e cateterismo suprapúbico (3,8\%) (2).

Assim é possível ver no estudo (3), que a maioria dos idosos utilizavam fraldas como forma de controle urinário, nos levando a buscar mais achados voltados para incontinência urinária que predispõe o indivíduo à infecções do trato urinário, ulceras de pressão e o aparecimento de dermatites perineais (4). Outra problemática encontrada na incontinência são as repercussões sobre o contentamento social e mental dos idosos, levando consigo isolamento social dependência e também a depressão.

De fato, não é possível esquecer, a importância de assinalar que a prevenção da DAF requer dos profissionais de saúde observação e vigilância constante do paciente, bem como a sistematização de cuidados para sua prevenção pela aplicação de protocolos preestabelecidos para proteção da pele. Estes devem seguir recomendações baseadas em evidências científicas (5).

Além disso, os idosos merecem uma atenção especial por vários motivos. Começando pelas mudanças fisiológicas com o envelhecimento natural, a incontinência coexiste com outras condições de morbidades, mobilidade reduzida e deficiência cognitiva (6).

Neste contexto, a prevenção e manejo da DAF dependerá, não somente de recomendações de técnicas adequadas ou da proposição das medidas atuais e protocolos baseados em evidências. Dependerá também da incorporação da importância deste cuidado, à medida que se compreende seu significado, e claro, do empenho, rigor e cuidado na realização dessa atividade que, embora pouco complexa tecnicamente, por vezes é 
negligenciada, e pode eventualmente incorrer em maior risco de lesões de pele, destacandose aqui a DAF.

Reunindo todos esses argumentos, mais o fato de que segundo USP (2018) (7), em 2030, o Brasil terá a população mais idosa do mundo. Justifica-se realizar tal estudo, no qual busca analisar as tecnologias desenvolvidas para prevenção e terapia tópica da pele com dermatite na área das fraldas. Ressaltando-se aqui a importância da pesquisa para a ciência que em concordância com Margotti (2011) (8), apresenta, que quando mais técnicas são descobertas, mais capacidade o indivíduo tem acesso para desenvolver novos saberes. Essa cadeia do conhecimento contribui de forma significativa para o desenvolvimento da percepção do homem e para o progresso.

Segundo o autor (9), devido aos poucos estudos sobre o problema da DAF na população idosa, e pelo fato de que a maioria das vezes, as medidas de prevenção e cuidados serem realizadas pela equipe de enfermagem, observa-se a importância do conhecimento dos profissionais acerca das estruturas morfológicas e funções da pele, que é necessário para identificar precocemente alterações nos pacientes.

Rrecomenda-se a aplicação de produtos de barreira, pois são considerados elementos essenciais para a prevenção da DAI. Sabe-se que o uso de produtos de barreira para o cuidado com a pele é comum e durante muitos anos os cremes e pomadas a base de óxido de zinco são considerados os produtos tradicionais para a prevenção e tratamento das lesões de pele por umidade $(10,11)$.

Fazendo-se necessário destacar os ácidos graxos essenciais que promovem quimiotaxia (atração de leucócitos) e angiogênese (formação de novos vasos sanguíneos), mantêm o meio úmido, aceleram o processo de granulação tecidual, facilitam a entrada de fatores de crescimento, promovem mitose e proliferação celular, atuam sobre a membrana celular, aumentando a sua permeabilidade, auxiliam o desbridamento autolítico e são bactericidas para S. aureus. (12).

É importante salientar que na população idosa é frequente uma carência sub-clínica de nutrientes (13). Sugerindo adequado fornecimento de minerais e os oligoelementos que são usados como co-fatores por diversas enzimas em inúmeras funções celulares relacionadas à imunidade e cicatrização de feridas. Níveis adequados destes elementos são necessários para a formação do colágeno, remodelação e reparo tecidual (14).

A pesquisa em questão objetiva avaliar o uso tópico dos Ácidos graxos essenciais (Ages) e Oligoelementos no controle das dermatites da área das fraldas (DAF) em idosos de uma instituição de longa permanência de Fortaleza. 


\section{METODOLOGIA}

$\mathrm{O}$ presente projeto de pesquisa se refere a um trabalho de conclusão de curso de bacharelado em enfermagem. Trata-se de um estudo descritivo, com abordagem quantitativa e delineamento transversal. Sabese que os estudos descritivos possuem a finalidade de observar, registrar e analisar os fenômenos ou sistemas técnicos, sem, contudo, entrar no mérito dos conteúdos. Não podendo haver a interferência do pesquisador. É vista como um estudo de caso, onde após a coleta é realizada uma análise dos dados. (15).

Por sua vez, o estudo quantitativo é aquele que se coleta e analisa dados quantitativos sobre variáveis. Se faz necessário incluir evidências que podem ajudar na eficácia dos dados é vista com uma pesquisa empírico-descritiva. Os dados estão apresentados em tabelas, gráficos e textos. (16). Não menos importante, o estudo transversal é definido por uma pesquisa observacional, realizada em um determinado espaço de tempo. (17)

O estudo envolveu 27 idosos de uma instituição de longa permanência especializada na assistência de pessoas idosas no período de setembro a dezembro de 2020 . A Instituição atende aproximadamente 60 idosos, divididos na cidade de Fortaleza e na Caucaia, sendo preferencialmente mulheres acima dos 60 anos, entretanto, como a instituição acolhe idosos em que grande parte são abandonados, hoje tem-se mais 4 homens.
Ainda assim desses 27 idosos, institucionalizados na Cidade de Fortaleza, inseridos na pesquisa, apenas $97 \%$ contém algum tipo de incontinência, e que fazem a utilização de fraldas, resultando assim 27 idosos constituintes da amostra. Desse modo o tamanho amostral, considerando a prevalência de incontinência em idosos institucionalizados de $90 \%$, com margem de erro de $7 \%$, confiabilidade de $95 \%$, o tamanho amostral mínimo será 27 idosos, com 7\% para menos e 32 idosos representando os $7 \%$ a mais.

A coleta dos dados foi efetivada em outubro e novembro de 2020, após a provação do comitê de ética (CEP), em 21, outubro de 2020 possuindo o CAAE: 37736720.8.0000.9987 e Número do Parecer: 4.351.731. Para tanto, foi respeitada de forma integral a Resolução 466/2012 do conselho Nacional de Saúde. Todos os participantes obtiveram o Termo de Consentimento Livre e Esclarecido (TCLE), onde concordaram na participação, assinaram o documento em duas vias (uma via ficou com o pesquisador e a outra com a instituição participante). A população foi constituída pelos sujeitos residentes do local de estudo conforme descrito anteriormente e a amostra decorreu de pacientes que atenderam os seguintes critérios de inclusão e exclusão:

\section{Critérios de inclusão}

Foram incluídos participantes que continham todas as condições descritas a seguir: 
- Indivíduos idosos, homens e mulheres, com 60 anos ou mais que concordassem em participar da pesquisa por meio da assinatura do Termo de Consentimento Livre e Esclarecido (TCLE);

- Internados há pelo menos 24 horas nas enfermarias clínicas, cirúrgicas e instituições de longa permanência;

- Apresentassem incontinência urinária e/ou fecal.

- Usuários de Fraldas e com integridade tissular prejudicada, compatível com DAF;

\section{Critérios de exclusão}

- Pacientes que, embora incontinentes por definição, possuíssem as seguintes condições: uso de cateter vesical de demora, outra derivação urinária ou pacientes anúricos que apresentaram concomitantemente constipação intestinal, uso de colostomia ou outra derivação intestinal para eliminações intestinais (devido ausência de qualquer tipo de contato com agente irritante na área de fralda).

- Óbitos e transferências durante o estudo;

- Idosos que apresentaram reação alérgica aos produtos selecionados para emprego no estudo;

\section{Coleta de dados}

Após anuência do local de estudo e subsequente aprovação do Comitê de Ética em Pesquisa (CEP), a instituição realizada a pesquisa foi contatada por meio de ligação telefônica à supervisão, agendando a data de início. Antes de proceder a coleta de dados, semanalmente, foi solicitado ao enfermeiro da unidade, a relação dos pacientes internados para definição dos pacientes elegíveis conforme critérios de inclusão e exclusão. Após, os coordenadores da instituição serem abordados em reuniões e realizados esclarecimentos sobre os objetivos da pesquisa, e também a realização do teste para COVIDE-19, foi solicitado a assinatura do termo de consentimento livre e esclarecido (TCLE).

Os dados foram coletados por meio de um instrumento elaborado para esse fim, e preenchido pelo próprio pesquisador composto por dados sociodemográficos, aspectos clínicos e referente a cuidados com a pele - envolvendo controle para DAF (APÊNDICE 1) e por meio de consulta de prontuário, analisamos comorbidades dos pacientes, e a predisposição para dermatites, além do exame físico da região de fraldas (ano genital + nádegas+ região das coxas e abdome). $\mathrm{O}$ exame se fez necessário a utilização do dermatoscópio, com a capacidade de aproximação: 40x, que permite identificar os tipos de lesão de pele com maior precisão, reduzindo a necessidade de 
procedimentos caros e invasivos, como biópsias e incisões. Os exames físicos com a captura das imagens foram divididos em 2 dias, no primeiro dia 13 idosos e no dia seguinte mais 14 idosos.

Mediante o diagnóstico da integridade tissular prejudicada em área de fraldas, em complemento ao instrumento de coleta de dados, associado ao registro fotográfico digital das regiões de fralda de todos os pacientes com presença sugestiva de DAF, e mediante autorização da captura da imagem para registro preciso das condições da pele, foi possível indicar a terapia tópica equivalente para cada idoso.

\section{Riscos}

- Um dos riscos foram o emocional, pois foi necessário expor as regiões afetadas pela DAF, uma vez que podem afetar a região ano genital, abdome, nádegas e porção superior das coxas, contudo a equipe realizou a avaliação em um lugar mais reservado onde o idosos se sentissem confortáveis e resguardados;

- Reações alérgicas poderiam ocorrer no estudo, visto que caso acontecesse, seriam utilizados produtos na pele acometida pela Dermatite na Área das Fraldas, contudo a equipe de pesquisa estava preparada e capacitada para a eventualidade, além das pomadas a base de corticoides para a eventual reação.

\section{Benefícios}

- O benefício do estudo, é enfatizar aqui a ação de produtos já utilizados, entretanto em outras métodos. Onde nesta nova formulação e tecnologia enriquecidos com oligoelementos, mostra-nos que os ácidos graxos essenciais associado aos oligoelementos são cruciais na hidratação e reconstituição do tecido, apresentando resultado satisfatório na prevenção e tratamento da DAF;

- Outra contribuição, é a do custo benefício, visto que os produtos utilizados são bem acessíveis, já que o estudo realizado, comprova hidratação adequada e proteção da pele, prevenindo a DAF.

\section{Metodologia de análise de dados}

Inicialmente foi realizada uma revisão de literatura de abordagem quantitativa de 50 trabalhos incluindo artigos, Teses e Sites, porém apenas 30 foram relevantes para o estudo. Utilizou-se os acervos: Pubmed, Lilacs utilizando os descritores: Dermatite das Fraldas, incontinência urinaria e/ou fecal, idoso, Crescimento Demográfico com ênfase no envelhecimento e fisiopatologia da pele.

O tratamento dos dados, foram através do lançamento desses elementos na planilha Microsoft Excel, com digitação e aplicação de fórmulas, variáveis quantitativas que estão representadas por meio de, teste t, 
exato de Fisher e em seguida teste t Studant, afim de obter médias e desvios-padrões, além de identificar incongruências. Para análise de dados subjetivos do instrumento referente aos dados, como a divisão de grupos, possuidores de DAF, os dados foram analisados em gráficos e análise estatística descritiva.

\section{Aspectos Éticos da Pesquisa}

Todos os cuidados éticos que tratam de pesquisas com seres humanos foram observados e respeitados, segundo a Resolução $n^{\circ}$ 466/12. Os responsáveis pelos idosos após a leitura, compreensão e esclarecimento de dúvidas, assinaram o TCLE, antes de proceder a coleta de dados. Nos casos de idosos com comprometimento cognitivo, em situação de substancial diminuição em sua capacidade de decisão, a assinatura foi obtida pelos seus representantes legais.

\section{Desfecho primário}

A presença da dermatite da área das fraldas (DAF), na qual sobe avaliação correta é possível definir um tratamento e cuidados aptos para a rápida melhora do paciente, dessa forma evitando complicações futuras.

\section{RESULTADOS}

Neste estudo procurou-se avaliar o uso tópico dos Ácidos graxos essenciais (Ages) e Oligoelementos no controle das dermatites da área das fraldas (DAF) em idosos de uma instituição de longa permanência de Fortaleza. Para isso, foi necessário que fossem idosos a partir de 60 anos, residentes da instituição, apresentassem incontinência urinária e/ou fecal e que utilizassem fraldas com integridade tissular prejudicada, compatível com DAF. A amostra foi composta por 27 idosos com idades entre 60 e 91 anos, sendo a maior parte deles (87\%) do sexo feminino. Realizado o estudo no período de 25 de setembro de 2020 a 26 de novembro de 2020 .

A respeito das comorbidades encontradas, 6 comorbidades foram identificadas na amostra, das quais, alguns pacientes possuíam pelo menos 2: (30\%) Alzheimer, (22\%) Demência + HAS, (14\%) Alzheimer +HAS, (14\%) Depressão, (7\%) Ansiedade, (7\%) Parkinson, (3\%) Depressão + Parkinson, (3\%) HAS (Gráfico 1). 
Gráfico 1 - Distribuição das comorbidades dos idosos residentes da instituição $(27$ idosos $=100 \%)$. Fortaleza (CE), 2020

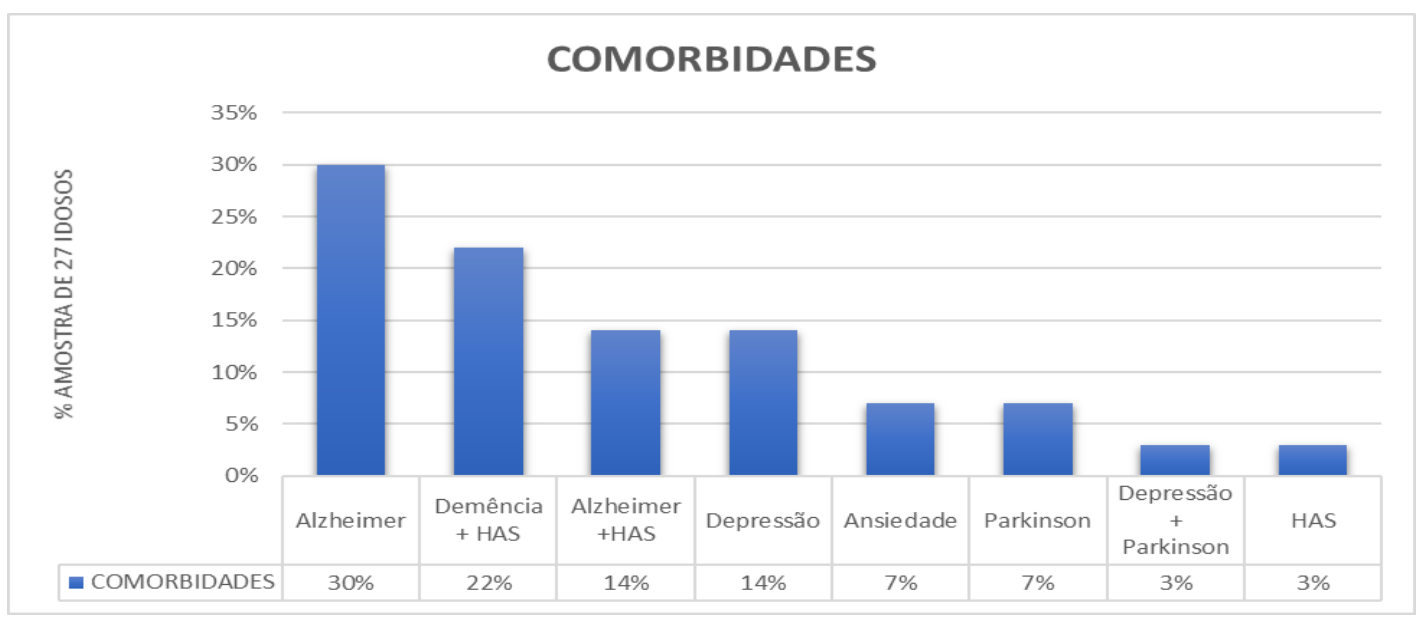

Fonte: Arquivos do pesquisador, 2020

Em relação a presença de incontinência, as variáveis eram de idosos que continham incontinência urinária $(4,15 \%)$, média de $0,1481 \ldots$ em seguida pela incontinência urinária e fecal $(23,85 \%)$, com média de 0,8518..., foram observados 27 idosos, para isso utilizou-se teste $\mathrm{t}$, para que a média de uma amostra possa medir a probabilidade da média da amostra (Tabela 1). Vale destacar que a amostra é de 27 $(100 \%)$ dos idosos que estavam alocados na instituição. Os exames foram realizados afim de identificar a DAF, em 2 dias, a cada dia, um grupo de idosos foram alocados em um ambiente privado e com o dermatoscópio a pele foi visualizada e capturada imagens das lesões. A segunda variável fora relacionada as regiões analisadas como: região ano genital + nádegas $(7,26 \%)$, região ano genital $(7$, $26 \%)$, região ano genital + porção superior das coxas $(10,37 \%)$, nádegas + abdome + região ano genital $(3,11 \%)$.

Tabela 1- Distribuição dos idosos de acordo com a presença de incontinência urinária ou urinária/fecal com evidência de Teste-T de Studant $(27$ idosos = 100\%). Fortaleza $(\mathrm{CE}), 2020$.

Teste-t: duas amostras em par para médias

\section{Incontinência urinária e Incontinência fecal urinária}

Média

0,851851852

0,148148148

Variância

0,131054131

0,131054131

Observações 


\section{Percentual \%}

$85 \%$

$15 \%$

Fonte: Arquivos do pesquisador, 2020

Os pacientes foram alocados em três grupos: $(21,75 \%)$ idosos tinham DAF (Figura $1 \mathrm{~A}),(5,22 \%)$ dos idosos não tinham DAF e $(1,3 \%)$ tinha placas fúngicas (Figura $1-B$ ). Sendo assim, após a avaliação da pele com o dermatoscópio, foram divididos novamente, agora em apenas 2 amostras: os que iram utilizar terapia tópica com a loção oleosa (12, 57\%), (AGE-ÓLEO). E a amostra que utilizou a terapia tópica com creme Hidratante (AGE-HIDRATANTE) $(9,43 \%)$.

Figura 1 - Captura de imagens de dois pacientes. A - Idoso com DAF. Região ano genital. Imagem ampliada em 40x. B - Idoso com fungos superficiais. Região ano genital + nádegas. Imagem ampliada em 40x
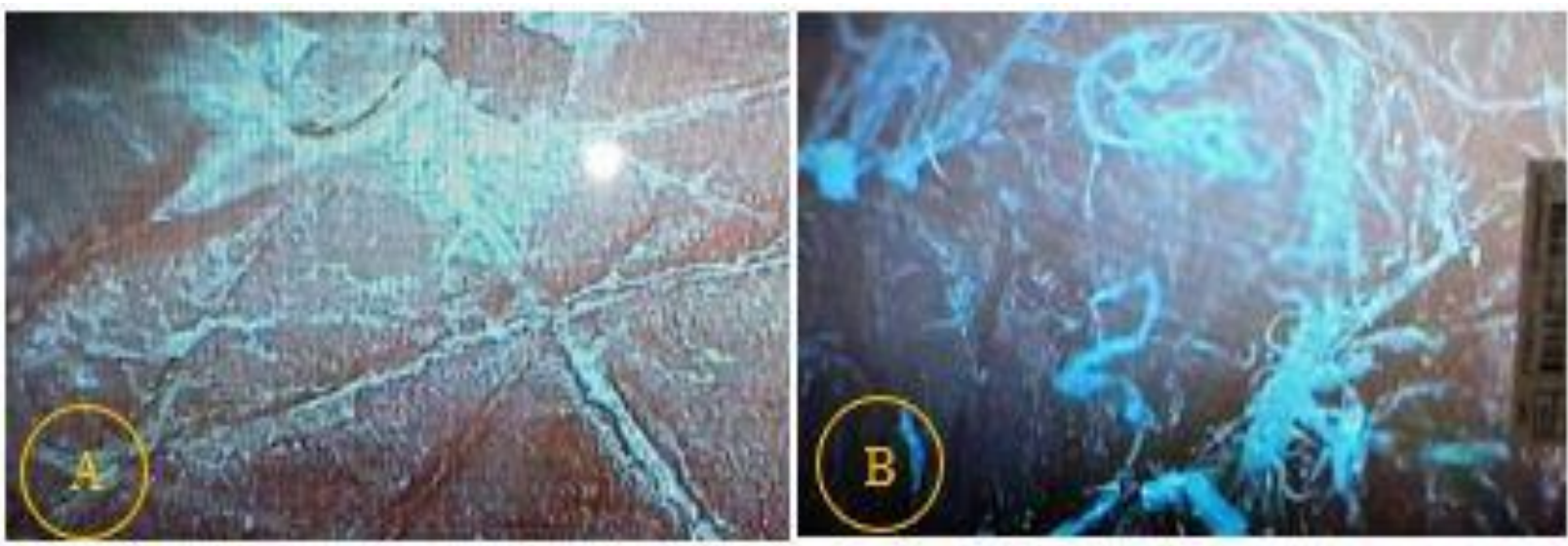

Fonte: Arquivos do pesquisador, 2020

Figura 2 - Evolução e Tratamento da DAF.
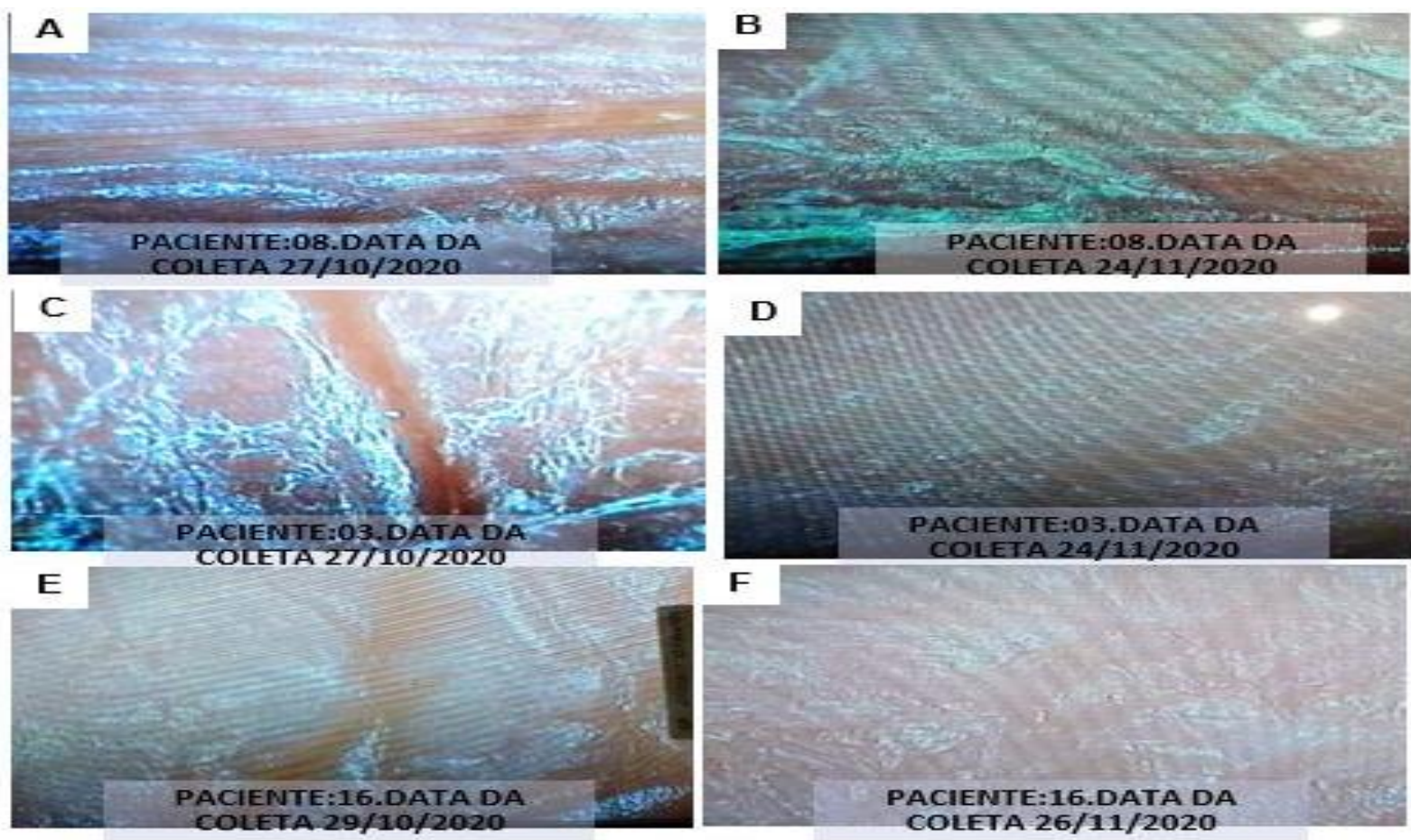
Imagem ampliada em 40x. A, p.08, primeira análise da pele, região ano genital + nádegas, submetida ao uso da loção oleosa (AGE-ÓLEO). B, p.08, participante da amostra, que fez o uso da loção oleosa (AGEÓLEO). apresenta reação negativa, exacerbando a DAF. C, p.03 com tecido desidratado, descamativo e eritematoso, submetido ao uso da loção oleosa (AGE-ÓLEO) como intervenção. D, p.03, reação positiva, promovendo melhor hidratação e reversão da DAF. E, p.16 primeira análise da pele, região ano genital + nádegas, submetida ao uso do creme Hidratante (AGE-HIDRATANTE). F, p.16 reação 100\% positiva, promovendo melhor hidratação e reversão da DAF. Fortaleza (CE), 2020.

Fonte: Arquivos do pesquisador, 2020

Como critério de divisão separamos idosos possuidores de pele descamativa, dermatite e com baixa hidratação para utilizarem terapia tópica com a loção oleosa de ácidos graxos essenciais AGE- Óleo, proporcionando maior hidratação. E os possuidores de dermatite e eritema fizeram o uso do creme Hidratante AGEHIDRATANTE que contém ácidos graxos essenciais e vitaminas, ingredientes responsáveis por restaurar a integridade cutânea. Sua fórmula possui vitamina E, que conta com propriedades antioxidantes, cicatrizantes, além de ser hipoalergênico.

Em 10 dias, já houve uma redução significativa do tecido eritematoso, das pápulas salientes e na pele descamativa. Uma ótima evolução para os que utilizaram a terapia tópica com creme Hidratante, assim como a terapia tópica com a loção oleosa (AGE-ÓLEO), apesar de 3 idosos não se adaptarem a terapia apenas com a loção oleosa, desencadeando novas dermatites na área das fraldas, na região das nádegas ocasionado pelo excesso de calor, somado ao contato com o material da fralda.
Para acompanhar e observar a evolução de cada grupo, que foram submetidos ao AGE-ÓLEO e ao AGEHIDRATANTE, após 30 dias separamos novamente em 2 dias, onde 13 idosos, passaram por uma nova captura de imagens e os 14 idosos restantes no dia subsequente, afim de visualizar melhora na evolução ou algum tipo de reação contrária à intenção que o produto propõe (Figura - 2).

Dos idosos submetidos ao uso do AGE-ÓLEO, amostra de (12, 100\%), (9, 75\%) tiveram uma ótima evolução, hidratação e melhora do quadro, já os $(3,25 \%)$ desenvolveram reação contraria, que com o calor intenso da cidade de Fortaleza desencadeou uma dermatite intensa. Iniciando-se nesses 3 idosos terapias tópicas do óleo em conjunto com o hidratante, promovendo a melhora gradativamente. Contrastando com o AGE-HIDRATANTE, que teve um ótimo resultado, onde tivemos a amostra de $(9,100 \%),(9,100 \%)$ tiveram uma ótima adaptação e evolução, $(0,0 \%)$ de reação adversa (Gráfico 2). 
Gráfico 2 - Apresenta o percurso dos pacientes desde a sua elegibilidade, recrutamento, intervenção e computação do estudo. $(27$ idosos = 100\%). Fortaleza (CE), 2020.

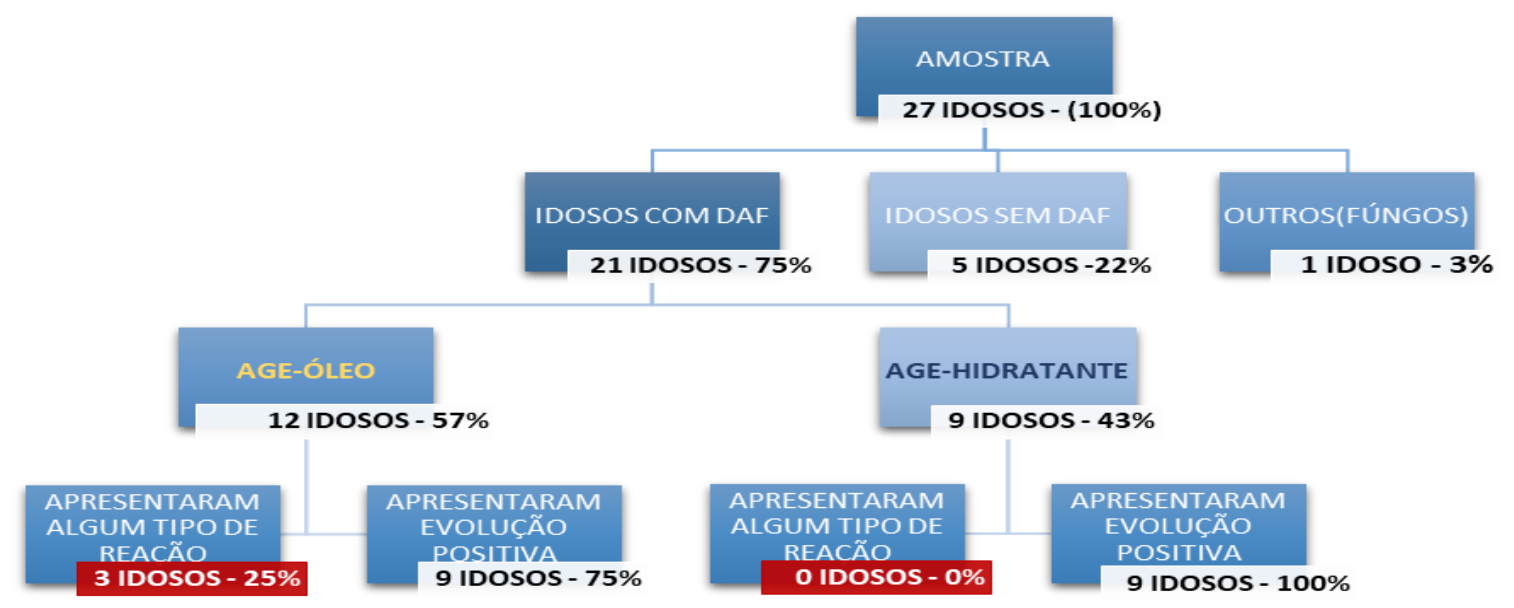

Fonte: Arquivos do pesquisador, 2020.

\section{DISCUSSÃO}

Os resultados demonstram através dos gráficos, tabelas e figuras, fatores importantes relacionados a DAF, suas características e manejo no tratamento tópico realizados na amostra. Estudos têm sido conduzido para demostrar melhor tratamento/prevenção da dermatite. As medidas de prevenção da DAF, na maioria das vezes, são realizadas pela equipe de enfermagem, entretanto em muitos casos não são realizadas com o objetivo de prevenção desse problema. $(1,24,27)$

Vale atentar uma série de condições expostas na pesquisa relacionados à idade onde, (24) afetam a eficiência da barreira da pele, notando-se um aumento do risco da DAF na pessoa idosa, simultaneamente, existe um maior risco de incontinência urinária e fecal nessa faixa etária, pesquisas revelam a associação entre a prevalência de
Incontinência Urinária e fatores de risco como doenças neurológicas, demostrado na amostra de 27 idosos, (30\%) Alzheimer, (22\%) Demência + HAS, (14\%) Alzheimer +HAS, (14\%) Depressão, (7\%)Ansiedade, (7\%)Parkinson, (3\%)Depressão + Parkinson, (3\%)HAS, portanto a incidência de DAF entre os idosos com comorbidades neurológicas são maiores, comparados aos idosos sem comorbidades neurológicas.

Na tabela - 1, através no Teste-t de studant: duas amostras em par para médias, mostrou que dos 27 idosos, $(23,85 \%)$ possuíam incontinência urinária e fecal, seguido dos que possuíam apenas incontinência urinária, $(4,15 \%)$, com uma média de 0,8518 , para incontinência urinaria e fecal, e 0,1481 para aqueles que continham apenas incontinência urinária. É sabido que as alterações na pele oriundas da DAF, (4) são desencadeadas por uma combinação de 
diversos fatores; sendo os mais significativos, o contato prolongado ou a irritação através da urina e fezes, e a maceração produzida pela umidade e o calor local. Sendo assim, o contato prolongado com a fralda molhada de urina e lípases fecais intensificam a probabilidade de desenvolver alterações da pele.

Atentando-se a isso, desenvolveu-se um estudo em que o protocolo de cuidado cutâneo incluía limpeza diária da área exposta à urina e fezes com água ou com água e sabão após cada episódio de evacuação, secagem cuidadosa da pele evitando o atrito e aplicação de um creme protetor e mesmo assim e apesar dos cuidados DAF ainda ocorreu. De acordo com a literatura, é importante destacar que a aplicação tópica de AGE reverte o ressecamento da pele, reduz a permeabilidade da mesma e corrige as composições anormais de ácidos graxos (19).

Existem diversos tipos de ácidos graxos, mas se tratando de tratamento de feridas, o ácido linoleico e o ácido linolênico são os mais importantes, pois não podem ser sintetizados pelos mamíferos, por não possuírem a enzima delta 9-dessaturase, posto isso, são chamados de ácidos graxos essenciais (AGE) (20). Consequentemente, a utilização do AGE na DAF, promove a proteção da pele das ações macerativas da umidade, diurese e fezes.

Importante destacar a ação do AGE com os Oligoelementos na proteção e cicatrização da pele, (14), os oligoelementos são usados como co-fatores por diversas enzimas em inúmeras funções celulares relacionadas à imunidade e cicatrização de feridas. Níveis adequados destes elementos são necessários para a formação do colágeno, remodelação e reparo tecidual. Defendendo sua influência no estudo, onde se objetiva avaliar seu uso tópico na DAF.

Destaca-se a importância do cuidado de enfermagem na avaliação minuciosa a técnica adequada na higiene, contribuindo diretamente na prevenção da DAF, visto que o enfermeiro é posto constantemente a atualizações dos cuidados e novas tecnologias nos tratamentos de dermatites e lesões. A vigilância da equipe de enfermagem nos idosos portadores de incontinência urinária e/ou fecal é primordial no desenvolvimento e melhora da DAF, considerando a anatomia e fisiologia do seu sistema tegumentar.

Os pacientes que foram alocados em três grupos: $(21,75 \%)$ idosos tinham DAF, $(5,22 \%)$ dos idosos não tinham DAF e (1, $3 \%$ ) tinha placas fúngicas, apresentados no (Gráfico 2). Aos pacientes que não tinham DAF continuaram com os cremes de barreira que já faziam uso na instituição, visto que não apresentaram alterações que levassem ao diagnóstico de DAF, seguindo apenas com orientações de higiene e troca de fraldas, os mesmos durante a pesquisa não apresentaram alterações na derme. Segundo os autores (21), a efetividade de intervenções não 
farmacológicas para o tratamento e prevenção do problema foi avaliada por alguns estudos, evidenciando que quando se intervém de maneira adequada e criteriosa, a incidência de danos causados pela DAF é reduzida.

Já o idoso com placas fúngicas, foi utilizado Nitrato de Miconazol dermatológico como terapia tópica. Visto que frequentemente surge infecções secundárias por Cândida. A instituição é vinculada com a Unidade de Atenção Primaria a Saúde, onde lá são realizados os acompanhamentos e encaminhamentos, caso necessário para a tenção especializada. Os possuidores de DAF que foram divididos em 2 amostras: AGE-ÓLEO e AGE-HIDRATANTE, obtivemos uma comparação significativa, onde no AGE-ÓLEO, 3 idosos tiveram reação contrária, apresentando DAF intensa, como intervenção foi utilizado o óleo + hidratante. Em contraste com AGE-HIDRATANTE onde 100\% apresentaram evolução positiva (Figura 2).

Essa diferença entre o AGE-ÓLEO e AGE-HIDRATANTE, foi bem pequena, visto que a eficácia das duas amostras foi favorável, e a composição dos mesmos serem semelhantes, onde a Lecitina de Soja e as Vitaminas A e E que contem neles proporcionam reparação tecidual. As vitaminas A e E possuem propriedades antioxidantes e protegem a membrana celular do ataque dos radicais livres. A Lecitina de Soja além de ser um agente de proteção, proporciona a manutenção da hidratação dos tecidos e ajuda no processo de cicatrização da pele (22). Assim, mudando apenas a apresentação e as diversas formas de utilização, apesar do AGE-ÓLEO, ter apresentado irritação na pele de 3 idosos, alguns fatores podem ter contribuído como: o excesso de produto, o calor excessivo na região das fraldas onde o plástico fica em contado, a demora nas trocas, e por esses pacientes serem acamados, e dificultar essa troca constante.

Os pacientes que apresentaram irritação, realizamos uma nova visita ao lar afim de averiguar o estado da pele, e pensar em uma nova estratégia para combatermos a DAF. Assim associamos o AGE - ÓLEO com AGE - HIDRATANTE no local, além de reduzir a quantidade de produto na região. Com isso, eles obtiveram uma melhora significante quanto ao primeiro momento de avaliação da pele com o Dermatoscópio.

\section{Já o AGE- HIDRATANTE,} apresentou melhor resolutividade na pele dos idosos, o uso do hidratante proporcionou absorção rápida da pele, com a atuação de agentes umectantes que permitiram uma hidratação profunda e o reestabelecimento da pele, assim revertendo a DAF. Sabemos que a região ano genital, nádegas, coxas e a utilização das fraldas por 24 horas na pele em contato com o plástico, favorecem o ressecamento e desenvolvimento de DAF, e com uma hidratação adequada e duradoura irá proporcionar o melhor conforto para esse idoso. 
Considerando nossos resultados em concordância com a literatura, os autores (23), exibe o comprometimento da elasticidade cutânea somada ao ressecamento proveniente da diminuição da secreção das glândulas sebáceas e sudoríparas leva a pessoa idosa a uma maior exposição e maior risco de rompimento cutâneo. Logo, a hidratação da pele é uma medida preventiva que ajuda a amenizar o ressecamento da pele, diminuindo o risco de lesões, como a DAF.

\section{CONSIDERAÇÕES FINAIS}

Em resposta aos objetivos do estudo, concluiu-se que, o uso tópico dos Ácidos Graxos Essenciais associados a oligoelementos proporciona prevenção e tratamento satisfatório, referente a integridade tissular prejudicada na área das fraldas, levando em consideração os principais fatores de risco da população idosa. Vale ressaltar que tanto o AGE-ÓLEO e AGEHIDRATANTE, são recomendados para a prevenção e tratamento da DAF, desde que avaliado a pele do paciente e as condições que o mesmo se encontra, como: se é acamado, deambula, hospitalizado, institucionalizado, número de trocas de fralda, como é realizado a higienização e a aplicação dos produtos.

A limitação do estudo, foi a ocorrência da pandemia (COVID-19), que diminuiu as visitas semanais, em apenas um dia por semana, porém o acompanhamento foi remoto com a enfermeira da instituição e as cuidadoras. O contado com o paciente idoso, foi reduzido, apenas um pesquisador poderia fazer as análises com o dermatoscópio. Lembrando que o pesquisador foi submetido ao exame do COVID-19, antes de entrar em contato com o idosos, reuniões foram acordadas, exigências e protocolos de higiene foram rigorosamente seguidos.

Assim, é importante desenvolver ações educativas, para aperfeiçoamento das práticas dos profissionais de saúde. Buscando avanços no conhecimento da temática, cabe destacar que mesmo com a escassez de evidências científicas, sobre a utilização do AGE e oligoelementos na prevenção e proteção da pele, não significa que essa formulação não tenha eficácia, por isso, se faz necessária realizações de mais ensaios clínicos, já que essa substância é muito utilizada no Brasil.

\section{REFERÊNCIAS}

1.Aquino Al, Chianca TCM, Brito RCS. Integridade da pele prejudicada, evidenciada por dermatite da área das fraldas: revisão integrativa. Rev. Eletr. Enf. [Internet].2012 abr/jun;14(2):414-24.

2. Bitencourt GR, Alves LAF, Santana RF. Practice of use of diapers in hospitalized adults and elderly: cross-sectional study. Rev Bras Enferm [Internet]. 2018;71(2):343-9. DOI: http://dx.doi.org/10.1590/0034-71672016-0341,2018.

3. Bitencourt GR, Santana RF. Instrumentos de avaliação dos eventos adversos associados ao uso de fraldas geriátricas. Rio de Janeiro. Rev Rene.v.20: e 39494, 2019. 
4. Silva J. Dermatite na área de fraldas em idosos internados em unidade de clínica médica. 2017;28. Trabalho Acadêmico (Bacharel em enfermagem) - Faculdade de Ciências da Saúde da Universidade de Brasília, 2017.

5. Gray M, Black JM, Baharestan MH, Bliss DZ, Colweel JC, Goldberg M, Kennedy-Evan $\mathrm{KL}$ et al. Moistureassociated skin damage: overview and pathology. Journal Wound Ostomy Continence Nursing. 38(3); 233-41. 2011.

6. Lucas MG, Bedretdinova D, Berghmans LC, Bosch JLHR, Burkhard FC, Cruz F et al. Guideline on urinary incontinence European Association of Urology, 75p. 2015.

7. Em 2030, Brasil terá a quinta população mais idosa do mundo. Jornal da USP. São Paulo.07-06-2018. Disponível em: https://jornal.usp.br/atualidades/em-2030brasil-tera-a-quinta-populacao-mais-idosa-domundo/. Acesso em: 25 de Setembro, 2020.

8. Margotti Lucas. A Importância do Trabalho Científico. Administradores.com.23-02-2011. Universidade Federal de São João Del Rei (UFSJ). Disponível em: https://administradores.com.br/artigos/aimportancia-do-trabalho-cientifico. Acesso em: 26, setembro de 2020.

9. Silva JLG, Faustino AM. Cuidados Relacionados à dermatite na área de fraldas em idosos hospitalizados. Revista de Enfermagem do Centro-Oeste Mineiro. 9(2721): 2019 .

10. Beeckman D. A decade of research on IAD: Evidence, knowledge gaps and next steps. Journal of tissue viability. Elsevier. p110. 2016.

11. Moreno Jimenez JC. Dermatitis del pañal. Salud Rural. X(9): 31-9. 1993.

12. Manhezi AC, Bachion MM, Pereira AL. Utilização de ácidos graxos essenciais no tratamento de feridas. Rev. Bras. Enferm. 2008;61(5):620-629.
13. Myers SA, Takiguchi S, Slavish S, Rose CL. Consistent wound care and nutritional support in treatment.Decubitus 1990; 3: 1628.

14. Bottoni A, et al. Papel da Nutrição na Cicatrização. Revista Ciências em Saúde V1, N 1, São Paulo. abril 2011.

15. Perovano, Dalton Gean. Manual de Metodologia Científica. Paraná: Editora Juruá; 2014.

16. Knechtel MR. Uma abordagem teóricoprática Metodologia da pesquisa em educação dialogada. Curitiba: Inter saberes, 2014.

17. Haddad MCFL. Qualidade da assistência de enfermagem - o processo de avaliação em hospital universitário público. Escola de Enfermagem de Ribeirão Preto. Tese de Doutorado. Ribeirão Preto, 2004. DOI: 10.11606/T.22.2004.tde-25112004-100935.

18. Beguin AM. et al. Improving diaper design to adress incontinence associated dermatites. Biomed Central. 2010; 10(86):

19. Protey C. Investigation of functions of essential fatty acids in the skin. $\mathrm{Br} \mathrm{J}$ Dermatol. 1977;97(1):29-38.

20. Mahan LK, Escot-Stump S. Krause: alimentos, nutrição e dietoterapia. $11^{\mathrm{a}}$ ed. São Paulo: Roca; 2005.

21. Fernandes JD, Machado MCR, Oliveira ZNP. Quadro clínico e tratamento da dermatite da área das fraldas - Parte II. Rev. An Bras Dermatol. 2009;84(1):47-54.

22. Ehrlich .P. Hunt TK. Effects of cortisone and vitamin A on wound healing. Ann Surg. 1999;167(3):324-8.

23. Freitas LDO, Waldman BF. O processo de envelhecimento da pele do idoso: PósGraduação em Enfermagem da Universidade Federal do Estado do Rio de Janeiro, 2017. 
24.Alcoforado CLGC. Efeito da pomada de óxido de zinco e da película de barreira não irritante na prevenção da dermatite associada à incontinência em idosos hospitalizados: Ensaio Randomizado.2018. 121p. Programa de Pós-graduação Stricto sensu - Doutorado em Enfermagem da Universidade Federal de Minas Gerais, como requisito parcial à obtenção do título de Doutor em Enfermagem. Belo Horizonte- MG, 2018.

25.Fernandes JD, Machado MCR, Oliveira ZNP. Quadro clínico e tratamento da dermatite da área das fraldas - Parte II. Rev. An Bras Dermatol. 2009;84(1):47-54.

26.Resende DM, Bachion MM, Araújo LA. Integridade da pele prejudicada em idosos: estudo de ocorrência numa comunidade atendida pelo Programa Saúde da Família. Rev. Eletrônica de Enfermagem. Acta Paul Enferm.[Internet] 200619(2):168-73.

27.Saurusaitis AD. Análise de custosminimização do cuidado com a pele na dermatite associada à incontinência. 2017.105 p. Programa de pós-graduação em enfermagem. (UNIRIO) Universidade Federal do Estado do Rio de Janeiro. Rio de Janeiro, 2017.

Submissão: 2021-05-16

Aprovado: 2021-09-06 